\title{
Present Status of Adjuvant Chemotherapy for Elderly Breast Cancer Patients
}

\author{
Sibylle Loibla ${ }^{a, b} \quad$ Mattea Reinisch ${ }^{a, c}$

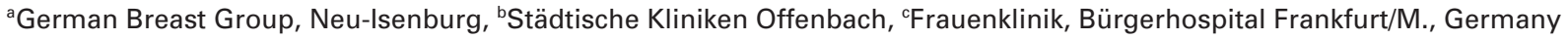

\section{Keywords}

Adjuvant chemotherapy - Elderly patients - Breast cancer

\section{Summary}

Elderly breast cancer patients are underrepresented in clinical trials, leading to a lack of knowledge regarding their tolerance of modern chemotherapy regimens. In addition, physicians are often reluctant to treat older patients with chemotherapy due to potential side effects. This article summarizes the up-to-date literature on chemotherapy in elderly patients with breast cancer, evaluates the impact of the patients' comorbidities and treatment alterations and aims to encourage treating patients adequately according to their disease in combination with the biological age rather than the chronological age alone. Finally, a short overview is given of the recruiting studies in Europe evaluating chemotherapy in elderly patients.

\section{Introduction}

More than $50 \%$ of breast cancers (BCs) are diagnosed in patients aged 65 years or older [1]. The BC incidence rises with increasing age, reaching a plateau between 65 and 69 years. The proportions and absolute numbers of BC cases and deaths in the elderly will increase due to increasing life expectancy. In addition, BC among the elderly will soon be a major public health concern, and treatment must be optimized with regard to efficacy, quality of life, and cost effectiveness.

\section{KARGER}

Fax +497614520714

Information@Karger.de

www.karger.com (c) 2012 S. Karger GmbH, Freiburg

$1661-3791 / 12 / 0076-0439 \$ 38.00 / 0$

Accessible online at:

www.karger.com/brc

\author{
Schlüsselwörter \\ Adjuvante Chemotherapie - Ältere Patientinnen . \\ Brustkrebs
}

\section{Zusammenfassung}

Brustkrebs-Patientinnen, die älter als 65 Jahre alt sind, werden häufig nicht in klinische Studien eingeschlossen oder repräsentieren nur einen sehr kleinen Teil der Studienpopulation. Daher ist das Wissen um das Ausmaß des Therapieerfolges in dieser Gruppe begrenzt, insbesondere bei Kombinationstherapien mit modernen und zielgerichteten Medikamenten. Zusätzlich sind die behandelnden Ärzte aus Angst vor den möglichen Nebenwirkungen häufig zurückhaltend, ältere Patientinnen mit Komorbiditäten mit zum Teil toxischen Medikamenten zu therapieren.

Dieser Artikel fasst die aktuelle Literatur zur Therapie von älteren Brustkrebs-Patientinnen zusammen, bewertet die Bedeutung der Komorbiditäten und möchte dazu ermutigen, die Patientinnen adäquat zum Krankheitsstadium und zur Tumorbiologie zu therapieren, ohne das chronologische Alter der Patientinnen zu stark zu bewerten. Zusätzlich fasst es die aktuell rekrutierenden Studien zur Therapie von älteren Patientinnen mit Brustkrebs zusammen.

A European study shows that the 5-year relative survival rates had improved significantly from 1990-1994 to 2000-2004 [2], although the difference was greater for patients younger than 70 years. In another trend analysis based on World Health Organization (WHO) mortality data, the median reduction of BC mortality from 1989 to 2006 was $37 \%$ for women younger than 50 years, $21 \%$ for those in the range of $50-69$ years, and only $2 \%$ for women older than 69 years [3].

Comorbidities including liver and kidney impairment need to be taken into consideration. It could be demonstrated that the quality of life in general decreases in all aspects measured [4]. 
Elderly patients have often been excluded from clinical trials investigating the advantages of new adjuvant systemic treatments. This has led to a lack of randomized controlled trials evaluating different chemotherapy regimens in elderly patients. The multimorbidity of elderly patients complicates the design of homogeneous study collectives. On the other hand, clinical trials designed for elderly patients had difficulties in recruitment (ACTION, ESTEEeM, and CASA trial), even with international collaboration [5]. Increasing age is significantly associated with low rates of chemotherapy [6]. Treatment recommendations for younger women cannot simply be transferred to elderly patients [7-9].

\section{Adjuvant Chemotherapy in Elderly Patients}

Adjuvant! Online is a tool for assessing the risk of recurrence and death of an individual patient within 10 years, when receiving specific treatment (on the basis of well-validated factors such as age, estrogen receptor (ER) status, number of involved axillary lymph nodes, etc.). These tools might support the decision on the most appropriate adjuvant treatment regimen (chemotherapy, endocrine therapy, or none); however, they are a decision aid and do not direct towards a specific treatment regimen. In general, a taxane-containing chemotherapy lowers the recurrence risk by one-third, independent of age, whereas the absolute benefit is smaller in elderly patients. Nevertheless, chemotherapy is still the standard of care for many women with BC, irrespective of age. Among cooperative clinical trials sponsored by the National Cancer Institute for early-stage BC, women $\geq 65$ years of age constitute only $18 \%$ of the participants, whereas they constitute $49 \%$ of the eligible pool of newly diagnosed cases. One study suggested physicians as a key barrier to enrolment of older women in clinical BC trials [10]. While there is a paucity of data for women over the age of 70, a meta-analysis by the Early Breast Cancer Trialists' Collaborative Group (EBCTCG) [11] clearly delineates the benefits of chemotherapy for women up to the age of 69 years. This is true for various comparisons, e.g., anthracycline-containing regimens versus no anthracycline or taxane-containing regimens versus no taxane. The number of women aged 70 or older is small and therefore confidence intervals (CIs) are large. In these analyses, ER-negative patients in the age group of 60-69 years who received chemotherapy demonstrated a proportional (absolute) risk reduction of $18 \%$ (4\%) in recurrence and $8 \%$ $(4 \%)$ in mortality. This proportional reduction was about half as great as that seen in women under the age of 50 , with patients in this latter age group demonstrating a 34\% (5\%) and $27 \%(5 \%)$ reduction in recurrence and mortality, respectively. For women aged 50-69 years, these benefits translate into an absolute gain in 10-year survival of $2 \%$ for node-negative disease and $3 \%$ for node-positive disease. However, even if patients above the age of 50 might benefit less than younger patients, they still benefit. In a large retrospective study conducted by Bouchardy et al. [12] in 407 patients aged $\geq 80$ years, $41.7 \%$ of deaths were directly attributable to BC. Correct management with surgery plus adjuvant treatment remarkably reduced the adjusted hazard ratio (HR) for BCrelated death to 0.1 (95\% CI 0.0-0.4) compared with supportive care alone, and increased the 5-year BC-specific survival to $90 \%$ compared to $51 \%$ with tamoxifen alone [8].

The first, and still the only published, prospectively randomized study in elderly patients of 65 years or older with median- to high-risk BC was undertaken by the Cancer and Leukemia Group B (CALGB) [13]. The study included 633 patients and compared the efficacy of standard chemotherapy (either cyclophosphamide, methotrexate, and fluorouracil (CMF) or doxorubicin plus cyclophosphamide (AC)) with the oral 5-fluorouracil prodrug capecitabine as monotherapy in women with stage I-IIIB early-stage BC. Overall, the study demonstrated a significantly better disease-free and overall survival for the group receiving the combination chemotherapy. In an unplanned subset analysis, patients with hormone receptor-negative disease derived the highest benefit from the combination therapy. However, 4 cycles of $\mathrm{AC}$ is no longer considered as a standard chemotherapy for BC [14], although it might be considered as a standard therapy for elderly patients. Taxanes are considered for all patients as standard of care, irrespective of the lymph node status $[12,15,16]$. It could be demonstrated that 4 cycles of docetaxel and cyclophosphamide (TC) reduce the risk of relapse and death independent of age and also in the group of women older than 65 years [17]. Currently, ongoing trials evaluate if TC given 6 times is superior or at least as good as an anthracyclinecontaining standard regimen like FEC-Doc (5-fluorouracil, epirubicin, cyclophosphamide followed by docetaxel) or ECDoc. None of the proposed regimens, apart from AC, has been tested in the elderly population. Therefore, it is not valid to extrapolate the data by Jones et al. [17] and declare TC as 'new' standard chemotherapy for elderly patients or patients with cardiac diseases, as TC was tested in neither of these groups. Standard regimens such as EC followed by paclitaxel weekly have a favorable toxicity profile and might therefore be the preferred choice as adjuvant chemotherapy for fit elderly women with an indication for adjuvant chemotherapy [9]. Taxanes can be given also to elderly patients, which was demonstrated by a retrospective analysis of 4 large, prospectively randomized trials [18]. A retrospective analysis by $\mathrm{Du}$ et al. [19] examined the effectiveness of adjuvant chemotherapy in 5464 women aged 65 or older with node-positive operable BC registered at the Surveillance, Epidemiology and End Results (SEER) database. They concluded that mortality was significantly reduced in women aged 65-69 years who received adjuvant chemotherapy compared to those who did not. However, chemotherapy failed to show a significant effect on mortality in women aged $\geq 70$. Du and colleagues [20] reviewed Medicare claims for chemotherapy-induced 
adverse effects in 35,000 women with stage I-IV BC. They reported an increase of hospitalization according to comorbidities, but not by age alone. The CALGB published data on the toxicity experienced by older and younger patients treated with adjuvant chemotherapy for node-positive BC. In total, 6642 patients were included in the analysis of 3 trials: a comparison of cyclophosphamide, doxorubicin, and fluorouracil in 3 dose schedules; cyclophosphamide and doxorubicin with or without paclitaxel and cyclophosphamide, doxorubicin, and paclitaxel every 2 versus every 3 weeks. In total, $7 \%$ of the patients were older than 65 years and only $3 \%$ were older than $70 \%$. The authors reported that, adjusted for treatment arm and study, increasing age was associated with an increased risk of grade 4 hematologic toxicity, but not for nonhematologic toxicity.

Dose-dense chemotherapy has not been proven in women older than 65 years [21]. Trastuzumab has also not been especially investigated in elderly patients. But a subgroup analysis from the Herceptin Adjuvant (HERA) Study did show an effect of trastuzumab independent of age [22].

As outlined above, elderly patients being at lower risk of relapse have to have additional risk factors, especially in patients with endocrine-responsive disease, to justify an adjuvant chemotherapy, e.g., high lymph node involvement, large tumors, grade 3 tumors, HER2-positive disease (see section ICE II). Several tools might be helpful, i.e., Adjuvant! Online, the 70-gene signature and the recurrence score, to estimate the relapse risk and the probable benefit of chemotherapy [23].

\section{Side Effects in Elderly Patients}

It has been shown that older women tend to be more interested in the side effects and the impairment of their quality of life than younger patients when choosing an adequate treatment [24]. It is therefore of great importance to carefully balance the risks and side effects against the benefit of a given regimen. The 'expert' recommendation is a strong determinant of receiving chemotherapy for BC. The CALGB [25] reported that the preference for chemotherapy is associated with greater odds of receiving chemotherapy. In addition, when the chemotherapy indication is less strong, higher rating of the physician-patient communication is an important determinant of use. In this cohort, most of the elderly women preferred an active role in their treatment decisions.

Acceptance of therapy does not differ among younger and older patients. However, older patients are less willing to compromise their quality of life and independence for potentially increased survival [26]. General health and functional independency are key compounds of quality of life in elderly patients and should be considered in finding informed decisions. Age is an independent risk factor for receipt of nonstandard BC therapies. Taking into account even comorbidity and recurrence risk, women 75 years or older are more likely to receive non-standard therapy [10, 27]. Factors contributing to non-standard therapy include race, cultural environment, socioeconomic status, social support, comorbidities, and physical barriers (e.g., sensory impairment and poor mobility).

In retrospective analyses from our institute, it has been shown that not only anthracyclines but also taxanes induce a greater range of side effects in patients $>65$ years of age than in younger patients [28]. Nevertheless, older patients were able to cope with the side effects and adequate dosing of chemotherapy was possible. Supportive treatment such as the primary use of granulocyte colony-stimulating factor (G-CSF) should be considered regularly, as recommended by the American Society of Clinical Oncology (ASCO) panel [29] and the European Organisation for Research and Treatment of Cancer (EORTC) guidelines [30], where the recommendation for the use of G-CSF has been expressed for all patients $\geq 65$ years when the planned chemotherapy has a risk of febrile neutropenia (FN) of $10 \%$ or higher. Age $\geq 65$ years is an individual risk factor for $\mathrm{FN}$.

\section{Adjuvant Chemotherapy Studies in Elderly Patients}

In Germany, the ICE trials (ibandronate and capecitabine in elderly patients) investigate the effectivity and safety of different therapy approaches in elderly patients with early BC.

The first ICE study (NCT00196859) is a prospective, multicenter, randomized phase III intergroup study comparing the outcome of patients of $\geq 65$ years with or without adjuvant capecitabine treatment [31]. Elderly patients with early BC with node-positive disease irrespective of additional risk factors or node-negative disease with at least 1 other risk factor (histologic tumor size $\geq 2 \mathrm{~cm}$, grade II or III, ER and progesterone receptor (PR) negative) received the bisphosphonate ibandronate with or without capecitabine. The primary endpoint was to compare disease-free survival between the 2 treatments.

The ICE study also analyzed the quality of life in elderly patients [32] receiving capecitabine with or without ibandronate and showed the expected difference in quality of life after the end of chemotherapy, although the physical functioning under capecitabine was not compromised. The global quality of life and the systemic therapy side effects were significantly higher in patients receiving capecitabine. This difference resolved soon after the end of the last capecitabine intake.

Capecitabine treatment is feasible in elderly patients with favorable hematologic and acceptable non-hematologic tolerability. Based on the observed safety profile in the first 100 patients, the ICE study therefore proceeded and finished recruitment in 2010 with close to 1500 patients. Early in 2013, after a median follow-up of 5 years, the analysis might be conducted. However, events are rare and the expected number of events has still not been reached. 
There is a need for improved taxane-based but anthracyclinefree combinations. It needs to be investigated whether anthracyclines in the adjuvant setting are necessary, especially in elderly patients who are at higher risk of cardiac toxicity, as anthracyclines can induce cardiac toxicity, especially congestive heart failure. This risk is increasing with age [33]. Jones et al. [17] could demonstrate that $4 \times \mathrm{TC}$ is as good as $4 \times \mathrm{AC}$. Based on the data by Muss et al. [13], the ICE II study compares the standard regimen of EC or CMF with an anthracycline-free regimen consisting of capecitabine and nanoparticle albumin-bound paclitaxel (nab-paclitaxel), to demonstrate superiority in terms of tolerability and efficacy of the anthracycline-free polychemotherapy.

The currently recruiting ICE II study (NCT01204437) is an investigational randomized phase II(-III) study in elderly $\mathrm{BC}$ patients at high risk of relapse based on either urokinasetype plasminogen activator/plasminogen activator inhibitor 1 (uPA/PAI-1) or a clinical-biological algorithm [34-36]. The ICE II study compares the disease-free survival for epirubicin plus cyclophosphamide (or CMF) with nab-paclitaxel plus capecitabine as adjuvant chemotherapy for elderly non-frail patients with an increased risk for relapse of a primary carcinoma of the breast. In case of a low-risk tumor, patients will not be included in the ICE II study.

Although therapy options for elderly patient started to receive more attention recently, the studies focusing on this age group are still too few when checking www.clinicaltrials.gov for further studies investigating the elderly population [37]. The National Cancer Institute of Italy currently runs a study in patients of 65 years to $<80$ years, comparing the disease-free survival when treated with CMF versus adjuvant weekly docetaxel. They report in an unplanned safety analysis [38] the side effects of these 2 different regimens, concluding that docetaxel has less hematologic side effects than CMF.

\section{Geriatric Assessment and Clinical Recommendations of Adjuvant Chemotherapy Regimens}

Elderly patients cannot only be discriminated by age alone. Increasing age is associated with a variety of changes like decreased organ function, increased comorbidity, comedication, decreased functional status, and limited life expectancy. Different tools are known to separate the fit elderly patients from the frail ones. A comprehensive geriatric assessment (CGA) bases the decision on dependencies in daily activities, comorbidities, depression, cognition, nutritional status, and social circumstances. A geriatric functional status measurement is different form the instruments used in oncology like the Karnofsky index or the Eastern Cooperative Oncology Group (ECOG) performance status. Extermann et al. [39] demon- strated that geriatric tools and oncology tools have only a weak correlation. Therefore, a geriatric assessment should be done in all elderly patients irrespective of their BC risk. There is currently no standard defined and no assessment tool routinely implemented in everyday clinical practice. But there is strong evidence in the general elderly population that routine implementation of geriatric assessment can improve patient compliance, treatment tolerability, quality of life, and finally also survival. The assessment focuses on comorbidities, depression, nutrition and activity of daily living, and the extent of restriction [40]. Especially older patients participating in a cancer clinical trial should have some kind of geriatric assessment (G8 score, Charlson score, Vulnerable Elders Survey (VES) score, instrumental activities of daily living (IADL)). The EORTC elderly task force for elderly patients suggests a 2-step approach: A short, simple screening tool could be used to identify the fit patients who do not need a full geriatric assessment. For the others, a more formal CGA is required (in collaboration with geriatricians) to provide a more complete view of the patient's status and to allow the identification of patients, who need special support and monitoring while receiving the chemotherapy, as they have an increased risk of toxicity.

In concordance with the EORTC, the German AGO (Arbeitsgemeinschaft Gynäkologische Onkologie) [41] also recommends the geriatric assessment of elderly patients. They suggest the Charlson comorbidity index [42] as a widely used tool with broad experience, as well as the G8 test. It is a screening tool in form of a questionnaire, which includes 7 Mini Nutritional Assessment items and 3 age ranges $(<80$, $80-85,>85$ years), for a total score ranging from 0 (poor score) to 17 (good score) [43].

Mild to moderate comorbidity does not seem to influence survival compared to patients with no comorbidities [44]. In general, older patients should not run the risk of undertreatment based on the fear of increased toxicity, decreased compliance, and increased hospitalization due to side effects. Geriatric assessment will help the physician weigh the risk of relapse or death from $\mathrm{BC}$ against comorbidities. Evidencebased treatment recommendations should be developed taking the patient's biological age into account and not only the chronological age.

The AGO [32] distinguished between the fit and the frail elderly patient with a life expectancy of more than 5 years or less, in addition to substantial comorbidities or not. A further interpretation of the comorbidities is up to the investigator. In case of a fit elderly patient $<70$ years old with a life expectancy of more than 5 years, the patient should be treated according to standard recommendations with regard to chemotherapy, hormonal therapy, and surgery. If the patient is $>70$ years old, administration of chemotherapy should be done within clinical trials. In frail older patients, a monochemotherapy should be preferred and surgical interventions 
should be done with caution. Nevertheless, adequate treatment according to the patient's situation and disease should not be withheld.

\section{Conclusions}

There is no aspect of management of older individuals with $\mathrm{BC}$ that should be driven by chronological age alone. A multidisciplinary oncological and geriatric approach may optimize the management of older individuals. Patient preference, comorbidities, and potential side effects should guide the management decisions. Patients should be closely monitored for side effects, with prompt intervention. Several BC trials in older individuals closed prematurely due to poor accrual. Currently recruiting clinical trials should be taken as an opportunity to further increase the evidence upon which we base our decisions.

The recommendations of the International Society of Geriatric Oncology (SIOG) and the European Society of Breast Cancer Specialists (EUSOMA) emphasize [10]: ‘... Despite competing causes of death, breast cancer is the cause of death in a substantial number of older patients. In women $\geq 80$ years at diagnosis, up to $40 \%$ die from breast cancer. Underestimation of life expectancy and fitness for therapy may result in age-related under-treatment, itself a risk factor for breast cancer recurrence and death.'

\section{References}

1 www.krebsdaten.de [last accessed July 30, 2012].

2 Rosso S, Gondos A, Zanetti R, et al.: Up-to-date estimates of breast cancer survival for the years 2000-2004 in 11 European countries: the role of screening and a comparison with data from the United States. Eur J Cancer 2010;46:3351-3357.

3 Autier P, Boniol M, La Vecchia C, et al.: Disparities in breast cancer mortality trends between 30 European countries: retrospective trend analysis of WHO mortality database. BMJ 2010;341:c3620.

$\checkmark 4$ Schwarz R, Hinz A: Reference data for the quality of life questionnaire EORTC QLQ-C30 in the general German population. Eur J Cancer 2001;37:1345-1351.

$\checkmark 5$ Reed MWR, Wyld L, Ellis P, Bliss J, Leonard R; ACTION and ESTEem Trial Management Group: Breast cancer in older women: trials and tribulations. Clin Oncol (R Coll Radiol) 2009;21:99-102.

6 Griggs JJ, Hawley ST, Graff JJ: Factors associated with receipt of breast cancer adjuvant chemotherapy in a diverse population-based sample. J Clin Oncol 2012;30:3058-3064.

$\checkmark 7$ Goldhirsch A, Glick JH, Gelber RD: Meeting highlights: International Consensus Panel on the Treatment of Primary Breast Cancer. J Natl Cancer Inst 1998;90:1601-1608.

8 Albrand G, Terret C: Early breast cancer in the elderly: assessment and management considerations. Drugs Aging 2008;25:35-45.

$\checkmark 9$ Biganzoli L, Wildiers H, Oakman C, et al.: Management of elderly patients with breast cancer: updated recommendations of the International Society of Geriatric Oncology (SIOG) and European Society of Breast Cancer Specialists (EUSOMA). Lancet Oncol 2012;13:e148-e160.

10 Kemeny MM, Peterson BL, Kornblith AB, et al.: Barriers to clinical trial participation by older women with breast cancer. J Clin Oncol 2003;21: 2268-2275.

-11 Early Breast Cancer Trialists' Collaborative Group (EBCTCG): Comparisons between different polychemotherapy regimens for early breast cancer: meta-analyses of long-term outcome among 100,000 women in 123 randomised trials. Lancet 2012;379:432-444.

12 Bouchardy C, Rapiti E, Gerald F, et al.: Undertreatment strongly decreases prognosis of breast cancer in elderly women. J Clin Oncol 2003;21:3580-3587.
13 Muss HB, Berry DA, Cirrincione CT, et al.: Adjuvant chemotherapy in older women with early-stage breast cancer. N Engl J Med 2009; 360:2055-2065.

14 Shen Y, Dong W, Feig BW, et al.: Patterns of treatment for early-stage breast cancers at the M. D. Anderson Cancer Center from 1997 to 2004. Cancer 2009;115:2041-2051.

15 Martin M, Pienkowski T, Mackey J, et al.: Adjuvant docetaxel for node-positive breast cancer. N Engl J Med 2005;352:2302-2013.

16 Martín M, Seguí MA, Antón A, et al.: Adjuvant docetaxel for high-risk, node-negative breast cancer. N Engl J Med 2010;363:2200-2210.

17 Jones S, Holmes FA, O'Shaughnessy J, et al.: Docetaxel with cyclophosphamide is associated with an overall survival benefit compared with doxorubicin and cyclophosphamide: 7-year followup of US Oncology Research Trial 9735. J Clin Oncol 2009;27:1177-1183.

18 Loibl S, von Minckwitz G, Harbeck N, et al.: Clinical feasibility of (neo)adjuvant taxane-based chemotherapy in older patients: analysis of $>4,500$ patients from four German randomized breast cancer trials. Breast Cancer Res 2008;10:R77.

19 Du XL, Jones DV, Zhang D, et al.: Effectiveness of adjuvant chemotherapy for node-positive operable breast cancer in older women. J Gerontol A Biol Sci Med Sci 2005;60:1137-1144.

20 Du XL, Osborne C, Goodwin JS: Population based assessment of hospitalizations for toxicity from chemotherapy in older women with breast cancer. J Clin Oncol 2002;20:4636-4642.

21 Moebus V, Jackisch C, Lueck HJ, et al.: Intense dose-dense sequential chemotherapy with epirubicin, paclitaxel, and cyclophosphamide compared with conventionally scheduled chemotherapy in high-risk primary breast cancer: mature results of an AGO phase III study. J Clin Oncol 2010;28:2874-2880.

22 Untch M, Gelber RD, Jackisch C, et al.: Estimating the magnitude of trastuzumab effects within patient subgroups in the HERA trial. Ann Oncol 2008;19:1090-1096.

23 Hartmann S, Gerber B, Elling D, Heintze K, Reimer T: The 70-gene signature as prognostic factor for elderly women with hormone receptorpositive, HER2-negative breast cancer. Breast Care (Basel) 2012;7:19-24.
24 Mandelblatt JS, Sheppard VB, Hurria A, et al.: Breast cancer adjuvant chemotherapy decisions in older women: the role of patient preference and interactions with physicians. J Clin Oncol 2010;28:3146-3153.

25 Mandelblatt JS, Faul LA, Luta G, et al.: Patient and physician decision styles and breast cancer chemotherapy use in older women: Cancer and Leukemia Group B protocol 369901. J Clin Oncol 2012;30:2609-2614.

26 Crivellari D, Aapro M, Leonard R, et al.: Breast cancer in the elderly. J Clin Oncol 2007;25:18821890.

27 Enger SM, Thwin SS, Buist DS, et al.: Breast cancer treatment of older women in integrated health care settings. J Clin Oncol 2006;24:4377-4383.

28 Linder M, von Minckwitz G, Harbeck N, Janni W, Kümmel S, Kaufmann M, Elling D, Nekljudova V, Loibl S: Comparison of the (non-)hematological toxicity and compliance of different anthracycline containing regimen with the standard $\mathrm{AC} / \mathrm{EC}$ in elderly patients with primary breast cancer. Cancer Res 2010;70(suppl):abstr P5-10-09, 387s.

29 Smith TJ, Khatcheressian J, Lyman GH, et al.: 2006 update of recommendations for the use of white blood cell growth factors: an evidence-based clinical practice guideline. J Clin Oncol 2006;24:31873205.

30 Aapro MS, Bohlius J, Cameron DA, Dal Lago L, Donnelly JP, Kearney N, Lyman GH, Weber DC, Zielinski C: 2010 update of EORTC guidelines for the use of granulocyte-colony stimulating factor to reduce the incidence of chemotherapy-induced febrile neutropenia in adult patients with lymphoproliferative disorders and solid tumours. Eur J Cancer 2011;47:8-32.

31 Reimer T, Nitz U, Potenberg J, et al.: ICE Study: A prospective, multi-centre, controlled, open-label, randomized phase III trial of ibandronate (I) with or without capecitabine $(\mathrm{X})$ in elderly patients (pts) with early breast cancer (GBG 32). Eur J Cancer 2009;7(suppl):215, abstr 4003.

32 Reimer T, Joel B, von Minckwitz G, et al.: Quality of life (QoL) in elderly patients (pts) with earlystage breast cancer treated with ibandronate (I) with or without capecitabine (X): results of the GBG 32 ICE trial. Eur J Cancer 2009;7(suppl):219, abstr 4016 . 
33 Pinder MC, Duan Z, Goodwin JS, et al.: Congestive heart failure in older women treated with adjuvant anthracycline chemotherapy for breast cancer. J Clin Oncol 2007;25:3808-3015.

34 von Minckwitz et al.: ICE II: An investigational randomized phase II study on epirubicin (E) plus cyclophospamide (C) (or CMF) versus nab-paclitaxel plus capecitabine (PX) as adjuvant chemotherapy for elderly nonfrail patients with an increased risk for relapse of a primary carcinoma of the breast. J Clin Oncol 2010;28(suppl 15):abstr TPS104

35 Jänicke F, Schmitt M, Pache L, et al.: Urokinase (uPA) and its inhibitor PAI-1 are strong and independent prognostic factors in node-negative breast cancer. Breast Cancer Res Treat 1993;24:195-208.

36 Harbeck N, Alt U, Berger U: Prognostic impact of proteolytic factors (urokinase-type plasminogen activator, plasminogen activator inhibitor 1 , and cathepsins B, D, and L) in primary breast cancer reflects effects of adjuvant systemic therapy. Clin Cancer Res 2001;7:2757-2764.

37 www.clinicaltrials.gov/ct2/results?term $=$ NCT00331097 [last accessed August 18, 2012].

38 Nuzzo F, Morabito A, De Maio E, et al.: Weekly docetaxel versus CMF as adjuvant chemotherapy for elderly breast cancer patients: safety data from the multicentre phase 3 randomised ELDA trial. Crit Rev Oncol Hematol 2008; 66:171-180.

39 Extermann M, Overcash J, Lyman GH, et al.: Comorbidity and functional status are independent in older cancer patients. J Clin Oncol 1998;16:1582-1587.

40 Pallis AG, Fortpied C, Wedding U, et al.: EORTC elderly task force position paper: approach to the older cancer patient. Eur J Cancer 2010;46:1502-1513.

41 www.ago-online.de [last accessed August 20, 2012].

42 Charlson ME, Pompei P, Ales KL, et al.: A new method of classifying comorbidities in longitudinal studies: development and validation. J Chronic Dis 1987; 40:373-383.

43 Soubeyran P, Bellera CA, Gregoire F, et al.: Validation of a screening test for elderly patients in oncology. J Clin Oncol 2008;26(suppl 20):abstr 20568.

44 Land LH, Dalton SO, Jensen MB, Ewertz M: Influence of comorbidity on the effect of adjuvant treatment and age in patients with early-stage breast cancer. Br J Cancer 2012, in press. DOI: 10.1038/bjc.2012.472. 